\title{
A Novel Integration Scheme Based on Mean Shift and Region-Scalable Fitting Level Set for Medical Image Segmentation
}

\author{
PeiRui BAI ${ }^{1, a}$, Dandan SONG ${ }^{1}$, Lijun $\mathrm{BI}^{1}$, Lei $\mathrm{LI}^{1}, \mathrm{Tao}_{\mathrm{QI}}^{2, \mathrm{~b}}$ \\ ${ }^{1}$ College of Electronics, Communication and Physics, Shandong University of Science and \\ technology, Qingdao 266590, China \\ ${ }^{2}$ School of Information Engineering, Wuhan University of technology, Wuhan 430070, China \\ aemail:bprbjd@163.com, bemail:qt133@163.com
}

Keywords: Medical Image Segemntation; Mean Shift; Level Set; Integration Scheme

\begin{abstract}
In this study, a novel integration scheme for coupling the results of mean shift with initial contours of the region-scalable fitting level set method (RSF model) is presented. There are two main contributions in the study. First, a new adaptive threshold formula to fit dynamic range of the mean shift clustering results is proposed. Second, a double-side mapping mode is presented to improve the robustness of initialization. Experimental results demonstrate the adaptability and robustness of the proposed method, and accurate segmentation could be obtained for medical images.
\end{abstract}

\section{Introduction}

Medical image segmentation is a fundamental task in object recognition and assistance diagnosis. Due to different imaging mechanisms and display modalities, tough challenges need to be dealed with for biomedical image segmentation. For example, in order to handle the intensity inhomogeneity of MRI images or the speckle noise of ultrasound images, more attention should be taken into account to obtain reasonable segmentation results. In recent years, active contour models (ACMs) have become very popular in medical image segmentation[1]. In general, the ACMs can be categorized into two basic models, the edge-based models and the region-based models. Level set method, which is also called geometric active contours, is an important branch of ACMs. The central idea of level set methods is to represent a curve as the zero level set of a higher-dimensional function, and deform the curve controlled by a partial differential equation of the level set function. The advantages of level set method over the other related methods exist in its capability of modelling arbitrary shape, flexible topology merging and splitting, and ease modification to suit high-dimensional case [2-4].

For the edge-based level set methods, image gradient and optional balloon force are utilized to drive the curve towards boundaries[5,6]. The edge-based methods could capture desirable boundaries with good qualities if the initial contours placed at a suitable location. Unfortunately, most medical images often have obvious noise or weak edges. Therefore, preprocessing such as denosing or bias correction is necessary to avoid failing segmentation.

In contrast, the region-based level set methods are much less sensitive to noise or weak edges than the edge-based level set methods because they exploit statistical information inside and outside the contour. Several representative region-based level set methods have been developed in recent years. Chan-Vese level set method (CV model) is a popular global region-based variational level set method which utilizes global statistical information to identify object boundaries[7,8]. However, Due to the global nature of the average intensities, the CV model is limited to deal with images with intensity inhomogeneity. To address this issue, some classical local region-based level set methods were presented aiming to utilize effectively local statistical information and combine the information into an energy minimization framework[9-12]. The RSF model is a classical which can cope with intensity inhomogeneity effectively by extracting local information through a Gaussian kernel function with controllable scale parameter[9]. Other similar local region-based methods can be seen in [10,11,12]. However, it is found that the sensitivity of initialization and controlling parameters is still a common 
limitation for these local region-based level set methods. Try-and-cut initialization is usually needed when an unknown image is segmented. Therefore, it is attractive to develop methods guiding automatically the placement of initial contours.

In practice, much effort have been done to obtain appropriate initial contours by employing different methods such as thresholding or clusering techniques. Gibou et al has made a connection between the CV model and the K-Means clustering, and constructed a new hybrid K-Means level set algorithm by setting initial contours as a signed distance function. However, the method needs the time-consuming periodical re-initialization[13]. Li et al has proposed a new fuzzy level set algorithm which utilizes the results of the spatial fuzzy clustering to guide a fast level set formulation[14]. Gharipour et al has presented a new framework which combines the fuzzy C-Means clustering and the $\mathrm{CV}$ model[15]. It uses region-based and topological characteristics to guide membership function estimation in fuzzy C-Means clustering. However, due to the fact that same pixels may belong to different classes simultaneously in the fuzzy C-Means clustering, it is not ease to interpret the final segmentation results of complex objects.

In our previous work, a novel region-based level set method (MS-RSF level set method) by integrating the mean shift clustering with the RSF model have been proposed[16]. The MS-RSF model can obtain appropriate initialization by mapping the clustering results of mean shift to a binary step function. The advantage of mean shift over the K-means clustering or the fuzzy C-means clustering is that it can determine the class number automatically without any prior knowledge. However, the original MS-RSF model has still two limitations. First, when mapping the clustering results of mean shift to a binary step function, the fixed threshold value may cause mismatch between dynamic range of the clustering results and the binary step function. Second, it is usually limited to adapt the complex and changeful images by using the single-side thresholding mode. In this study, we address mianly these limitations. First, a new formula for estimating the adaptive threshold value is proposed to enhance the adaptivity of the mean shift clustering. Second, we extend the single-side thresholding mode to the double-side thresholding mode to improve the robustness of the method.

The remainder contents are organized as follows. In section II, we describe the background of the proposed integration scheme. In section III, details of the scheme are presented. Experimental results are presented in section IV. Concluding remarks are drawn in section V.

\section{Background}

\section{The RSF Level Set Method}

The level set method originates from the theory of wave propagation along interface of solid and liquid. Malladi et al has introduced it into the area of image processing[4]. The level set method represents the planar curve implicitly as the zero level set of a smooth Lipschitz function, and models the curve evolution by a partial differential equation of the level set function. Let $\phi(x, y, t)$ denotes the level set function, where $x, y$ is space variable and $t$ is time variable. The PDE equation of traditional level set method can be expressed as follows:

$$
\left\{\begin{array}{c}
\frac{\partial \phi(x, y, t)}{\partial t}=-F|\nabla \phi| \\
\phi(x, y, 0)=\phi_{0}(x, y)
\end{array}\right.
$$

where $|\nabla \phi|$ is gradient modulus of the level set function. It points toward normal direction of the active curves. F denotes speed of the evolution which depending on the curvature of contours. $\phi_{0}(x, y)$ denotes the initial contours at $\mathrm{t}=0$. Originally, a signed distance function and complex numerical iteration upwind scheme are used to solve the PDE equation. By adopting variational mechanism, the edge-based or the region-based level set methods can be diverted into a energy minimization framework which can incorporate additional information and be conducted through simple numerical implementation. For example, the CV model and the RSF model are two representative variational level set methods. 
The RSF model is a novel region-based level set method which is powerful to cope with intensity inhomogeneity[9]. The key idea is to extract local intensity information by approximating local image intensities on two sides of the contours. The scale controllable energies are then incorporated into an variational energy minimization framework. The partial differential equation of the RSF level set method can be expressed as follows:

$$
\left\{\begin{array}{c}
\left.\frac{\partial \phi(x, y, t)}{\partial t}=-\delta_{\varepsilon}(\phi)\left(\lambda_{1} e_{1}-\lambda_{2} e_{2}\right)+v \delta_{\varepsilon}(\phi) \operatorname{div}\left(\frac{\nabla \phi}{|\nabla \phi|}\right)\right]+\mu\left[\Delta \phi-\operatorname{div}\left(\frac{\nabla \phi}{|\nabla \phi|}\right)\right. \\
\phi(x, y, 0)=\phi_{0}(x, y)
\end{array}\right.
$$

The Dirac delta function $\delta_{\varepsilon}(\phi)$ is defined as follows:

$$
\delta_{\varepsilon}(x)= \begin{cases}0 & |x|>\varepsilon \\ \frac{1}{\pi} \frac{\varepsilon}{\varepsilon^{2}+x^{2}} & |x| \leq \varepsilon\end{cases}
$$

where $\varepsilon$ is a positive constant. There are three terms on the right side of (2).

The first term is a local data-fitting energy to drive the active contours towards object boundaries. The locality of region energy is contorlled by a Gaussian kernel function defined as follows:

$$
G_{\sigma}(u)=\frac{1}{(2 \pi)^{n / 2} \sigma^{n}} e^{-\frac{|u|^{2}}{2 \sigma^{2}}}
$$

where $\sigma$ is a positive parameter. $e_{i}(\mathbf{x}), i=1,2$ denotes the local fitting energies outside and inside the closed contour. $\lambda_{i}, i=1,2$ are their weighted coefficient respectively. The local fitting energies can be defined as follows:

$$
e_{i}(\mathbf{x})=\int G_{\sigma}(\mathbf{y}-\mathbf{x})\left|I(\mathbf{x})-f_{i}(\mathbf{y})\right|^{2} d \mathbf{y}, i=1,2
$$

where $I: \Omega \rightarrow R$ denotes a given image, $\Omega$ denotes image domain. $f_{i}(\mathbf{y}), i=1,2$ denotes weighted average of intensities in the neighborhood of each pixel.

The second term is arc length term which can maintain regularity of the evolving contour. The third term is regularization term to maintain regularity of the level set function. The parameters $v$ and $\mu$ are weighted coefficient respectively. Given an appropriate initial contour, the local energies will drive the active contours toward real boundaries under the constraint of smoothness and regularization.

\section{The MS-RSF Level Set Method}

The MS-RSF level set method first extracts global statistical information by the mean shift, then the initial contours obtained from the clustering results evolved under the constraint of the RSF model. According to the integration strategies introduced by Muñoz et al[17], the integration scheme of the MS-RSF level set method can be considered as a post-processing integration of the global and the local region information. The critical process is how to couple effectively the clustering results of mean shift with initial contours of the RSF model. Due to the intrinsic characteristic of variational level set method, the RSF model adopts a simple binary step function as its initial contours:

$$
\phi_{0}(x, y)= \begin{cases}-D & (x, y) \in \Omega_{0}-\partial \Omega_{0} \\ 0 & (x, y) \in \partial \Omega_{0} \\ D & (x, y) \in \Omega-\Omega_{0}\end{cases}
$$

where $\Omega_{0}$ is a subset in $\Omega, \partial \Omega_{0}$ denotes the points on the boundaries. D is a positive integer. To overcome the initialization sensitivity of the RSF model, we utilize clustering results of the mean shift to guide the placement of initial contours[18,19].

Let $C_{K}(x, y)$ represents clustering result of the mean shift with values from 1 to $\mathrm{K}$ ( $\mathrm{K}$ is a positive integer denoting the number of classes), The key problem is how to design a map process from 
$C_{K}(x, y)$ to $\phi_{0}(x, y)$. In our previous work, we design an integration scheme for completing the mapping procedure as the following two operations:

First, a simple thresholding process is conducted to convert the clustering results $C_{K}(x, y)$ into a binary image $B(x, y)$ :

$$
B(x, y)=\left\{\begin{array}{lc}
1 & C_{K}(x, y) \geq T \\
0 & \text { other }
\end{array}\right.
$$

where $T$ is a fixed threshold value with default value of 1.5 .

Second, a mapping process coverting $B(x, y)$ to a binary step function is conducted:

$$
\phi_{0 \_ \text {old }}(x, y)=-6(0.5-B(x, y))
$$

Through the above mapping process, the MS-RSF level set method can obtain appropriate initial contours automatically and reasonable results in most cases. We denote the operations in (7) and (8) as the old integration scheme. It is find that the old scheme has two main limitations when coping with complex medical images. First, when the clustering results of mean shift have large dynamic range, the fixed $T$ in (7) may cause failing results. Second, the single-side thresholding mode in (7) may reduce robustness of the MS-RSF level set method.

\section{The proposed integration method}

In this study, we propose a new adaptive threshold formula and a double-side thresholding mode to overcome the limitations of the MS-RSF level set method. The new integration scheme includes three steps.

First, a new formula is presented to obtain an adaptive threshold value by taking into account dynamic ranges of the clustering results:

$$
T(K)=\left\lfloor\frac{\left[\min \left(C_{K}(x, y)\right)+\max \left(C_{K}(x, y)\right)\right]}{2}\right\rfloor
$$

where $\lfloor x\rfloor=\max \{n \in z \mid n \leq x\}$ expresses rounding-off operation. By keeping the arithmetic mean value of $C_{K}(x, y)$ as integer, an adaptive threshold value changed with $\mathrm{K}$ can be estimated to fit the dynamic range of clustering results. By replacing the fixed threshold value $T$ in (7) with the adaptive threshold value $T(K)$ in (9), the adaptability of the MS-RSF level set method could be improved significantly.

Second, a new double-side thresholding mode is presented as follows:

$$
\begin{aligned}
& \text { case } 1: B(x, y)= \begin{cases}1 & C_{K}(x, y) \geq T(K) \\
0 & \text { other }\end{cases} \\
& \text { case } 2: B(x, y)=\left\{\begin{array}{lc}
1 & C_{K}(x, y)<T(K) \\
0 & \text { other }
\end{array}\right.
\end{aligned}
$$

Due to the diversity of medical images, it is usually a challenging task to obtain satisfied segmentation. Typical challenges such as blur or weak edges, complex contour shapes and overlapping objects etc may fail any simple image segmentation methods. It is found that the single-side thresholding mode in (7) may cause unstable evolution for images with low contrast-to-noise ratio (CNR). Therefore, we propose a complementary optional scheme to enhance the robustness of the method. That is, after obtaining $T(K)$, we first test the availability of case 1 in (10). If it is capable of obtaining reasonable results, case 1 is believed to be the optimal choice. Otherwise, case 2 is preferable to be adopted. In terms of our observation, this alternant choice can enhance the robustness obviously.

Third, a symmetrical binary step function can be obtained from following equation:

$$
\phi_{0 \_ \text {new }}(x, y)=-4 \alpha(0.5-B(x, y))
$$


where $\alpha$ is a weight coefficient with default value 1.0 . By submitting $\phi_{0_{-} \text {new }}(x, y)$ into (2), an improved MS-RSF level set method can be obtained as follows:

$$
\left\{\begin{array}{c}
\left.\frac{\partial \phi(x, y, t)}{\partial t}=-\delta_{\varepsilon}(\phi)\left(\lambda_{1} e_{1}-\lambda_{2} e_{2}\right)+v \delta_{\varepsilon}(\phi) \operatorname{div}\left(\frac{\nabla \phi}{|\nabla \phi|}\right)\right]+\mu\left[\Delta \phi-\operatorname{div}\left(\frac{\nabla \phi}{|\nabla \phi|}\right)\right. \\
\phi(x, y, 0)=\phi_{0 \_ \text {new }}(x, y)
\end{array}\right.
$$

We denote the operations in (9), (10) and (11) as the new integration scheme. Let $L\left(\phi_{i, j}^{k}\right)$ represents the driving force in the right hand side in (12), the proposed method can be implemented numerically as follows:

$$
\phi_{i, j}^{k+1}=\phi_{i, j}^{k}+\tau \cdot L\left(\phi_{i, j}^{k}\right)
$$

where $\tau$ represents time step.

\section{Experiments and discussion}

All the methods in this study are implemented in the R2008 version of MATLAB on a PC (Lenovo-M4600) with a 3.0 GHz Pentium(R)4 processor and 1.25GB RAM. The operating system is WindowsXP (Sercive Pack2). In the implemetation of the mean shift clustering, all pixels are marked with a positive integer within bandwidth of the center pixel, and the class would be merged if the difference between means of the classes is less than half of the bandwidth. The details of mean shift can be found in [18,19]. The parameters $\varepsilon$ in (3), $\alpha$ in (11) and $\mu$ in (12) are set to its default value 1.0 .

\section{Improvement of the New Integration Scheme}

To evaluate the improvement of the new integration scheme, we compare the results of the MS-RSF level set method adopting the old and new integration scheme. Three real medical images i.e. the cell.bmp (first row), phantom.jpg (second row) and vessel.bmp (third row) are used to illustrate the performance. The sizes are $83 \times 65$, 205×205, $111 \times 110$ respectively. Different challenges such as blur or weak edge, heavy noise and intensity inhomogeneity etc are posed by these images. In Figure 1 , the first column shows the original images. The second column shows the clustering results of mean shift. The class numbers for the three images are 5, 2, 3 respectively, which are determined automatically by the mean shift. The third column shows the final contours of the method using old integration scheme. The fourth column shows the final contours of the new integration scheme. Both methods adopt same controlling parameters which are listed in Table 1 . The final contours are poltted as red lines.

By visual inspect, the old method obtain failing results shown in Figure 1 (c)(g)(k) for all the three images. Especially, the result shown in Fig.1(c) completely lost the boundaries of objects. In contrast, successful results (Figure.1(d)(h)(l)) are obtained by the proposed method. All the successful results are obtained by employing the case2 in (10). Therefore, it is clear that the proposed method can provide more reliable driving forces to match different dynamic ranges and contrasts of the clustering results. In addtion, we can draw some simple rules for determining the key controlling parameters by implementing the tuning process. It is found that iamges with heavy noise or blurring edges need larger $\sigma$ and $v$. The larger $h$ is, the smaller $K$ is obtained.

\section{Comparison with the global and local region-based level set methods}

It is well known that the region-based level set methods are much less sensitive to initialization than the edge-based level set methods[12]. However, when dealing with medical images with complex background or heavy noise, the dirving forces posed by simple region information maybe insufficient. In this section, we compare our method with the typical global region-based CV model in $[7,8]$ and local region-based LIF model in [11].

Figure 2 shows the results of the CV model and the LIF model with a rectangular and circular binary initial contour respectively. It can be seen that failing results are obtained by the two models. The weak and blurring edges in cell.bmp and heavy noise in phantom.jpg make it hard to converge to 


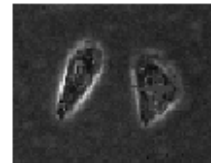

(a)

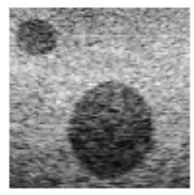

(e)

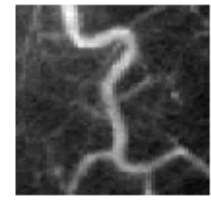

(i)

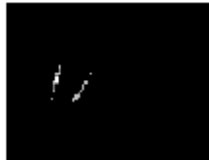

(b)

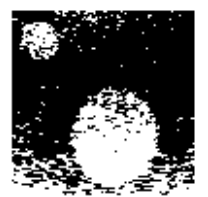

(f)

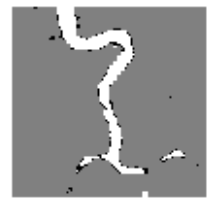

(j)

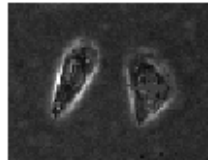

(c)

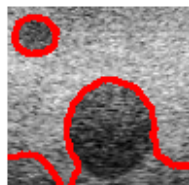

(g)

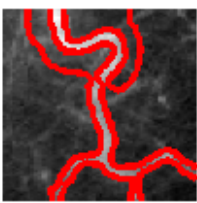

(k)

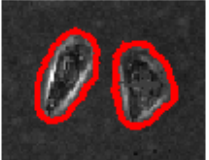

(d)

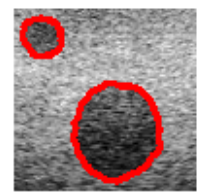

(h)

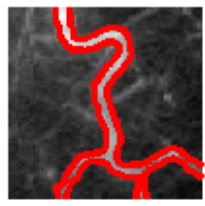

(1)

Fig.1 Results of the old and new MS-RSF level set method. First column: original images. Second column: mean shift clustering results. Third column: final contours of the old method. Fourth column: final contours of the proposed method. The final contours are poltted as red lines.

Table1 The controlling parameters of the results in Figure 1

\begin{tabular}{|l|c|c|c|c|c|c|}
\hline Test Image & $\boldsymbol{h}$ & $\lambda_{1}$ & $\lambda_{2}$ & $\sigma$ & $v$ & $\tau$ \\
\hline Cell.bmp & 15.0 & 2.5 & 1.0 & 20.0 & 0.01 & 0.2 \\
\hline Phantom.jpg & 12.0 & 1.0 & 1.75 & 15.0 & 0.115 & 0.2 \\
\hline Vessel.bmp & 6.0 & 1.0 & 1.0 & 3.0 & 0.001 & 0.1 \\
\hline
\end{tabular}

The genuine boundaries for the two models. The global nature of the average intensities of the CV model make it incapable of handling the images with obvious intensity inhomogeneity. Computation efficiency of the LIF model is attractive, but the sensitivity to the location, shape, size of initial contours may limit its application.

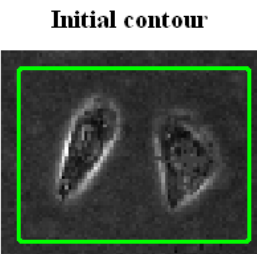

(a)

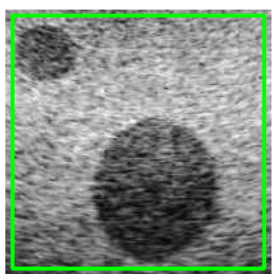

(g)
CV model

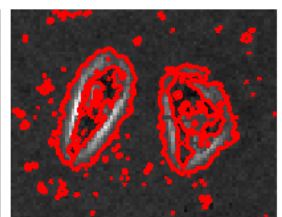

(b)

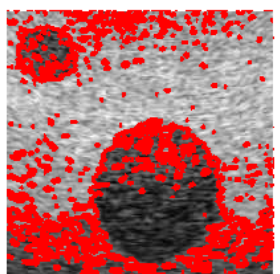

(h)

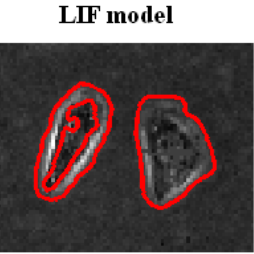

(c)

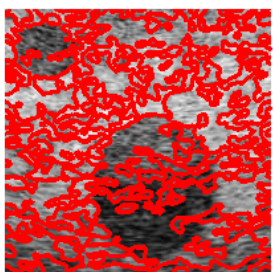

(i)

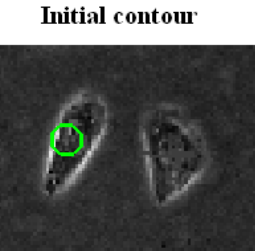

(d)

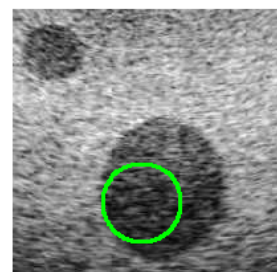

(j)

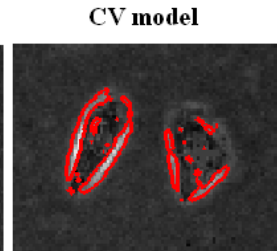

(e)

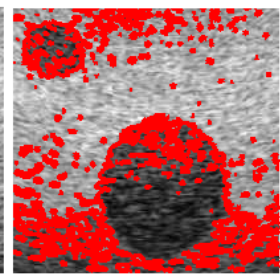

(k)

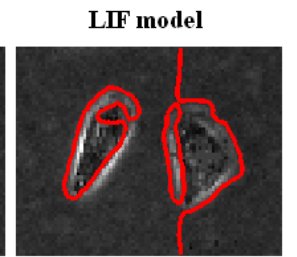

(f)

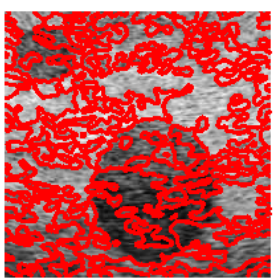

(I)

Fig.2 Results of the CV model and the LIF model with different initialization. First row: results of the cell.bmp with a rectangular and circular binary initial contour. Second row: results of the phantom.jpg with a rectangular and circular binary initial contour.The initial and final contours are poltted as green and red lines respectively. The iterative number is 1000 .

Figure 3 shows the results of the new method proposed in this study. The first and second columns show the original images and clustering results of the mean shift. The third column shows the initial contours (green lines) obtained from the new integration scheme. The fourth column shows the final contours plotted as red lines. It could be observed that with the initial contour mapped from the 
clustering results automatically, the proposed method could obtain successful results for all the three images. The advantages of the proposed method lies in three aspects. First, it eliminates completely the need of manual initial contour. More appropriate region close to the genuine boundaries could be obtained from the clustering results of mean shift. Second, it provides a novel pattern to utilize both global and local image information complementarily, and better performance can be obtained through this kind of combination. Third, the automaticity and robustness of the proposed method is higher than the traditional level set methods. It avoids the time-consuming try-and-cut process for choosing appropriate initialization, and can save the time for determining optimal controlling parameters.

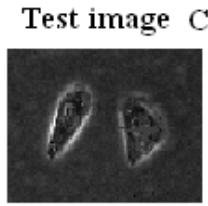

(a)

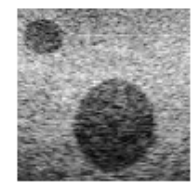

(e)

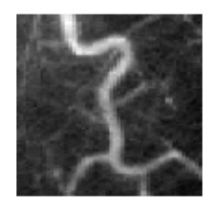

(i)

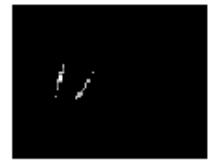

(b)

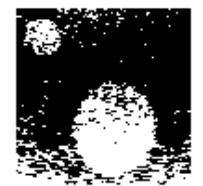

(f)

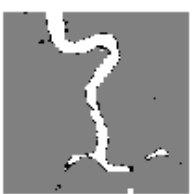

(j)

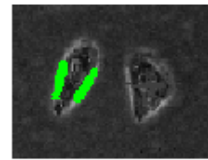

(c)

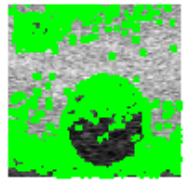

(g)

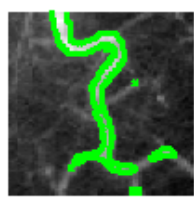

(k)

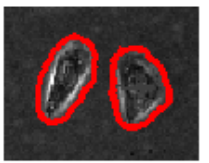

(d)

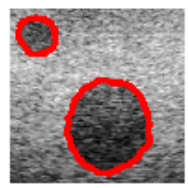

(h)

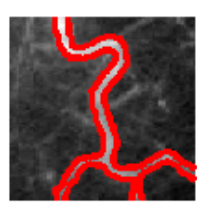

(1)

Fig.3 Results of the proposed method. First column: original images. Second column: clustering results of the mean shift. Third column: initial contours obtained from the new integration scheme. Fourth column: final contours of the proposed method. The initial and final contours are poltted as green and red lines respectively.

\section{Conclusion}

A novel integration scheme based on the mean shift clustering and the RSF level set method is presented. By considering how to match effctively with the dynamic range and contrast of the clustering results, a novel adaptive threshold formula and a double-side mapping mode are proposed to develop a new MS-RSF level set method. Experimental results confirm the improvements of adaptbility and robustness of the MS-RSF level set method adopting the novel integration scheme. It is noted that there are some novel methods have been introduced more recently to enhance the accurancy of local information extraction[20-22], so it maybe an interesting issue to introduce our scheme to these methods.

\section{Acknowledgment}

In this study, the research was supported by the National Natural Science Foundation of China (under Grant No.61471225 ) and the Project of Shandong Province Higher Educational Science and Technology Program (under Grant No.J13LN16). The authors would also like to thank Prof. ChunMing Li and Dr.KaiHua Zhang for sharing the code of RSF model and LIF model.

\section{References}

[1] L.He, Z.G.Peng, B Everding, X. Wang, C.Y. K.Han, L Weiss, W.G Wee, A comparative study of deformable contour methods on medical image segmentation [J], Image and Vision Computing, 2008 (26)141-163. 
[2] M.Amar, B.A Ismail, Variational and level set methods in image segmentation. New York: Springer-Verlag, 2010, ch.2.

[3] D.Cremers, M.Rousson, R.Deriche, A review of statistical approaches to level set segmentation: integrating colors, texture, motion and shape [J], International Journal of Computer Vision, 2007 (72)195-215.

[4] R Malladi, J.A Sethian, B.C Vemuri, Shape modeling with front propagation: a level set approach [J], IEEE Trans on Pattern Analysis and Machine Intelligence, 1995 (17)158-175.

[5] V.Caselles, R.Kimmel, G.Sapiro, Geodesic active contours [J], International Journal of Computer Vision, 1997 (22)61-79.

[6] C.M Li, C.Y Xu, C.F Gui, M.D Fox, Level set evolution without re-initialization: a new variational formulation [C], in 2005 IEEE Proc. Computer Society Conference on Computer Vision and Pattern Recognition, San Diego, 2005. 430-436.

[7] T.Chan, L.Vese, Active contours without edges [J], IEEE Trans on Image Processing, 2001(10) 266-277.

[8] T.Chan, L.Vese, A multiphase level set framework for image segmentation using the Mumford and Shah model [J], International Journal of Computer Vision, 2002(50)271-293.

[9] C.M.Li, C.Y.Kao, J.C.Gore, Z.H.Ding, Minimization of region- scalable fitting energy for image segmentation [J], IEEE Trans on Image Processing, 2008(17)1940-1949.

[10]S.Lankton, A.Tannenbaum, Localizing region-based active contours [J], IEEE Trans on Image Processing, 2008(17)2029-2039.

[11]K.H Zhang, H.H.Song, L.Zhang, Active contours driven by local image fitting energy [J], Pattern Recognition, 2010(43)1199-1206.

[12]L.He, S.F.Zheng, L.Wang, Integrating local distribution information with level set for boundary extraction [J], Journal of Visual Communication \& Image Representation, 2010(21)343-354.

[13]F.Gibou, R.Fedkiw, A fast hybrid K-means level set algorithm for segmentation [C], in 4th Annual Hawaii International Conference on Statistics and Mathematics, Hawaii, 2005.281-291.

[14]B.N Li, C.K.Chui, S.Chang, S.H.Ong, Integrating spatial fuzzy clustering with level set methods for automated medical image segmentation [J], Computers in Biology and Medicine, 2011(41)1-10.

[15]A.Gharipour, A.W-C.Liew, An integration strategy based on fuzzy clustering and level set method for cell image segmentation [C], in 2013 IEEE Conference on Signal Processing, Communication and Computing, KunMing, 2013.1-5.

[16]P.R.Bai, Q.Y.Liu, L.Li, S.H.Teng, J.Li, M.Y.Cao, A novel region-based level set method initialized with mean shift clustering for automated medical image segmentation [J], Computers in Biology and Medicine, 2013(43)1827-1832.

[17]X.Muñoz, J.Freixenet, X.Cufí, J.Martĺ, Strategies for image segmentation combing region and boundary information [J], Pattern Recognition Letters, 2003(24)375-392.

[18]D.Comaniciu, P.Meer, Mean Shift: a robust approach toward feature space analysis [J], IEEE Trans on Pattern Analysis and Machine Intelligence, 2002(24)603-619.

[19]P.R.Bai, C.H.Fu, M.Y.Cao, Y.Han, Improved mean shift segmentation scheme for medical ultrasound images [C], in 4th International Conference on Bioinformatics and Biomedical Engineering, Chengdu, 2010.1-4.

[20]Y.Wang, S.M.Xiang, C.H.Pan, L.F.Wang, G.F.Meng, Level set evolution with locally linear classification for image segmentation [J], Pattern Recognition, 2013(46)1734-1746. 
[21]Q.Zheng, E.Q.Dong, Narrow band active contour model for local segmentation of medical and texture images [J], Acta Automatica Sinica, 2013(39)21-30.

[22]L.F Wang, C.H Pan, Robust level set image segmentation via a local correntropy-based K-means clustering [J], Pattern Recognition, 2014(47)1917-1925. 